\title{
Improvement of AODV Routing on MANETs Using Fuzzy Systems
}

\author{
Taqwa Odey Fahad ${ }^{1}$, Prof. Abduladhim A. Ali ${ }^{2}$ \\ Department of Computer Engineering \\ University of Basra , Iraq \\ ${ }^{1}$ Taqwa.odey@yahoo.com , 2ªbduladem1@yahoo.com
}

\begin{abstract}
Most of routing protocols used for Mobile Ad hoc Network (MANET), such as Ad hoc on demand distance vector (AODV) routing, uses minimum hops as the only metric for choosing a route. This decision might lead to cause some nodes become congested which will degrade the network performance. This paper proposes an improvement of AODV routing algorithm by making routing decisions depend on fuzzy cost based on the delay in conjunction with number of hops in each path. Our simulation was carried out using $\mathrm{OMNeT++} 4.0$ simulator and the evaluation results show that the proposed Fuzzy Multi-Constraint AODV routing performs better than the original AODV in terms of average end-to-end delay and packet delivery.
\end{abstract}

Keywords : MANETs, multi constraint, traffic delay, AODV, fuzzy system.

\section{INTRODUCTION}

In recent years, the progress of communication technology has made wireless devices smaller, less expensive and more powerful. Such rapid technology advance has provoked great growth in mobile devices connected to the Internet. There are two variations of wireless network, which are infrastructure networks and ad hoc networks. In an infrastructure network, a mobile station must find the nearest base station within its communication range before it communicates with another, whereas an ad hoc network is a collection of mobile nodes that are capable of communicating with each other without the aid of any established infrastructure or centralized administration. They are selforganized, dynamically changing multi-hop networks as illustrated in figure (1).

Routing is the most fundamental research issue in MANET and must deal with limitations such as low bandwidth, high error rates and unpredictable movements of nodes. Generally, current routing protocols for MANET can be classified into three main categories according to the routing information updates mechanism [1] as proactive (table driven), reactive (on- demand), and hybrid. In proactive routing algorithms, each node must maintain path information from each node to the destination node and updates its routing table entries periodically, such as destination sequenced distance vector (DSDV)[2] and optimized link state routing (OLSR)[3]. Reactive routing protocols establish a route to a destination only when needed, the source node initiates a route discovery process when the route is required, and once a route has been established it is maintained until either the destination becomes inaccessible or until the route is no longer used, such as ad hoc on demand distance vector (AODV)[4], and dynamic source routing (DSR)[5]. Finally, in the hybrid routing approach the proactive and the reactive behaviors are mixed in amounts that match the efficient operation conditions for each class. Representative hybrid routing protocols include zone routing protocol (ZRP)[6], and zone-based hierarchal link state routing protocol (ZHLS)[7].

Recently, some intelligent methods including fuzzy systems have been applied to the area of routing in ad hoc networks, aiming to obtain more adaptive and flexible models over 
the existing models. However, in the relevant researches to this work [8-10] the destination node waits within a time frame for accepting all route request packets of the possible paths from the source, then it decides which is the suitable one after evaluating the cost of each path depending on fuzzy systems. In this work the routing wait time is reduced by making each node along the path from the source to the destination participating in selecting the optimal route, considering not only the minimum hops but also the delay period of each path using fuzzy systems assessment until reaching the destination which does not require any additional waiting time to send the route reply packet on the chosen path.

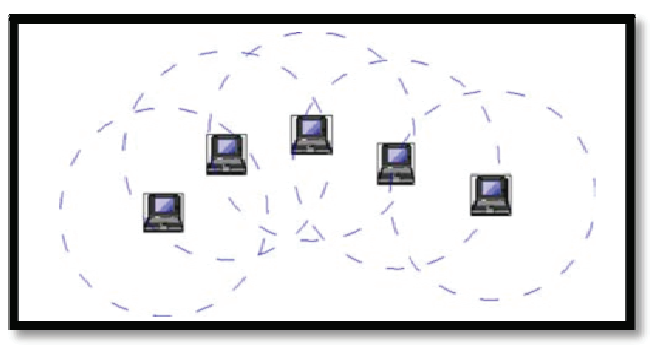

Figure 1: Mobile Ad hoc network

\section{FUZZY SYSTEM FOR FAODV}

Fuzzy logic theory [11][12] was first introduced by Zadeh in the 1960s as a tool for modeling the uncertainty of natural language. Fuzzy systems are suitable for uncertain or approximate reasoning, especially for the system with a mathematical model that is difficult to derive, and allow decision making with estimated values under incomplete or uncertain information. A fuzzy system as represented in figure (2) basically consists of basically three steps : fuzzification, inference engine, and defuzzification. Fuzzification is a process where crisp input values are transformed into membership values of the fuzzy sets. After the process of fuzzification, the inference engine calculates the fuzzy output using fuzzy rules which are linguistic in the form of if then rules. Defuzzification is a mathematical process used to convert the fuzzy output to a crisp value.

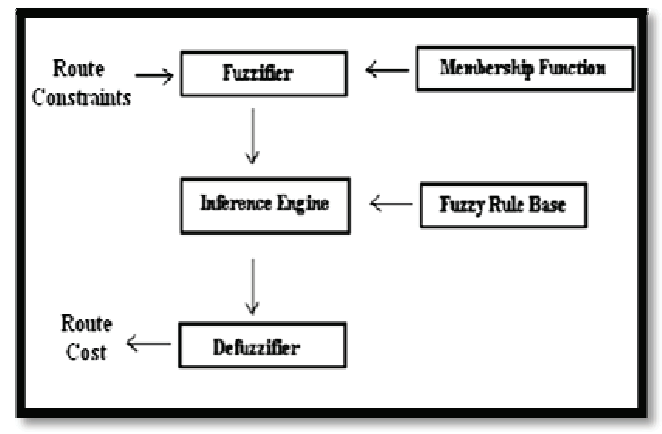

Figure 2 : Fuzzy Routing

In the proposed fuzzy system, Mamdani minimum inference method was used as the fuzzy inference method, where the 'and' operation was set to minimum and defuzzification was carried out using centroid defuzzifer.

As shown in figure (3), triangle membership functions were used to represent inputs and output, with three linguistic variables to the inputs : Low, Medium, and High, and five for the output: Very Low, Low, Medium, High, and Very High, while the fuzzy rule base is illustrated in table (1) .

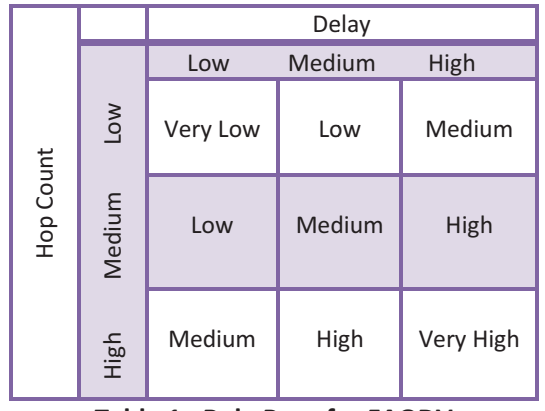

Table 1 : Rule Base for FAODV 


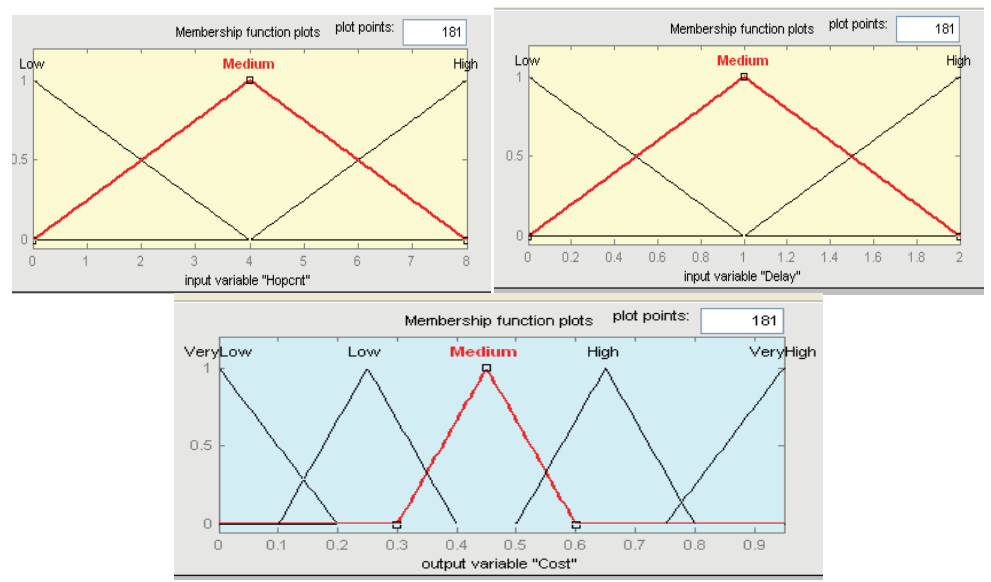

Figure 3: Membership functions of FAODV

\section{PROPOSED FAODV}

During the route discovery process of the AODV routing protocol, the Route Request Message (Rreq Msg.) carries the required fuzzy input parameters: Number of Hops and Delay taken along that path, each node embedded a fuzzy system which calculates the fuzzy cost each time it receives the Rreq Msg. and updates the reverse route entry to the source of the Rreq. Msg. if the new fuzzy cost less than the stored one, while the dissemination of the Rreq. Msg. is continued until getting the destination. In addition to reducing the routing time by this scheme, it also reduces the memory overhead required to store all routes until reaching the destination.

\section{SIMULATION MODEL}

Our simulation modeled a network of 20 / 30 mobile nodes placed randomly within $700 \times$ 700 meter area. Each node had channel capacity of 54 Mbps. The IEEE 802.11g was used as the medium access control protocol. A random waypoint mobility model was used with a speed ranging from $0 \mathrm{~m} / \mathrm{s}$ to $10 \mathrm{~m} / \mathrm{s}$. A traffic generator was developed to simulate CBR (Constant Bit Rate), UDP application. The size of the data payload was 512 bytes. Data sessions with randomly selected from eight sources and any other host as a destination were simulated. Each source transmitted data packets at a minimum rate of 2 packets/sec, and a maximum rate of 4 packets/sec. Each simulation was executed for 250 seconds of simulation time by OMNeT++ 4.0 simulator [13], the fuzzy system is implemented by $\mathrm{C}++$ language and added to the AODV Routing implementation provided by the INETMANET Framework on the chosen simulator.

\section{SIMULATION RESULTS}

We evaluated and compared the following metrics :

- Packet Delivery Ratio: is obtained by dividing the number of received data packets by the destinations by the number of data packets originated by the sources.

- Average End-to-End Delay: is the average time taken by data packets when released by sources until reach their destinations.

- Normalized Routing Load : is the total number of control packets (include Rreq, Rrep, Rerr, and Rrep_Ack packets) divided by number of transmitted data packets in the network. 
From the simulated results in figures (4-6), the packet delivery ratio is increased by about $5.07 \%, 4.96 \%$, also the average end-end delay is improved by about $49.517 \%, 45.326 \%$ for 20 , 30 nodes respectively with slightly improvement in the normalized routing load when using FAODV over the original AODV.

This results show that by utilizing fuzzy logic in the routing decision, better performance obtained.

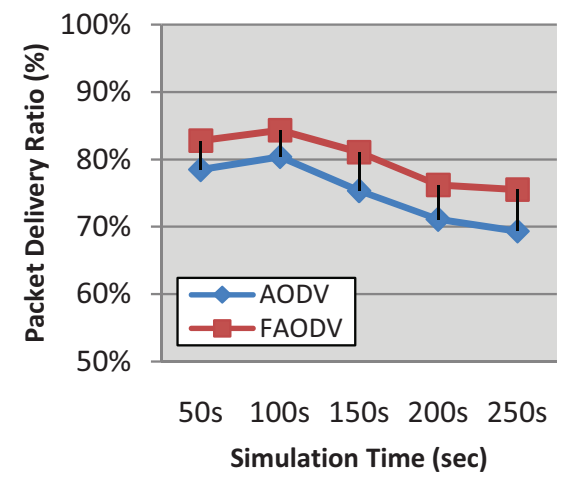

(a)

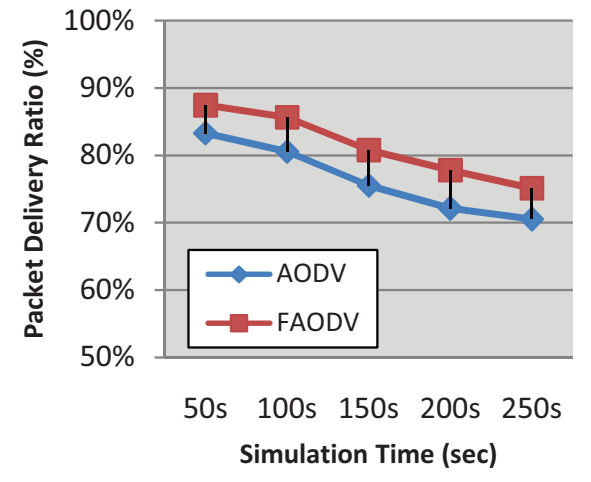

(b)

Figure 4: Packet Delivery Ratio Vs. Simulation Time:

(a): 20 nodes; (b): 30 nodes

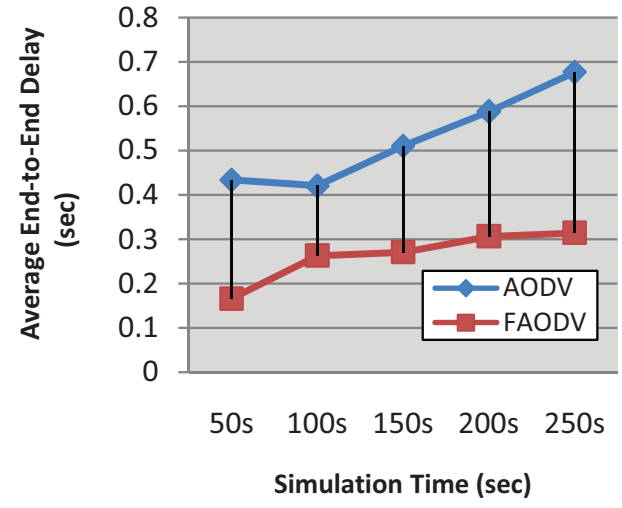

(a)

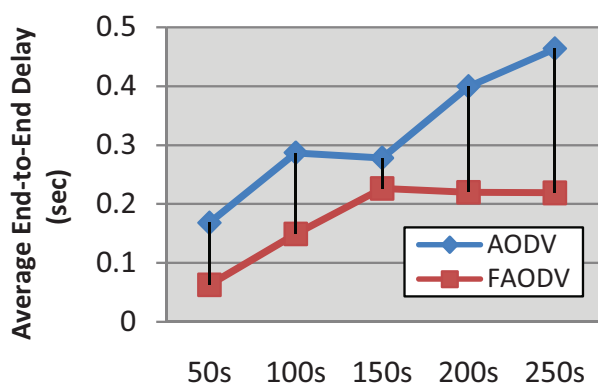

Simulation Time (sec)

(b)

Figure 5: Average End-to-End Delay Vs.

Simulation Time:

(a): 20 nodes; (b): 30 nodes

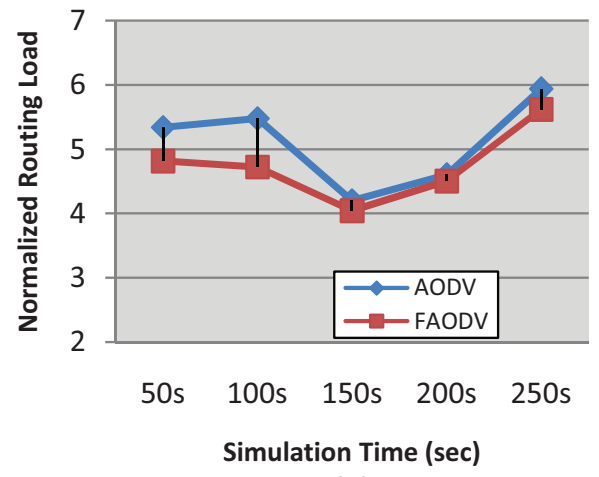

(a) 


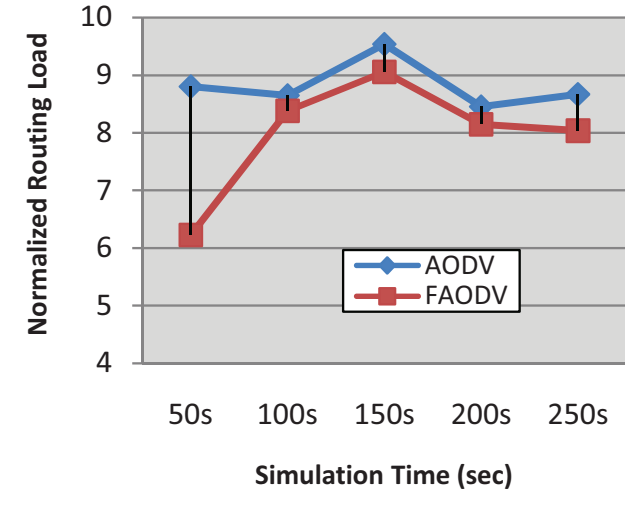

(b)

Figure 6: Average End-to-End Delay Vs. Simulation Time:

(a): 20 nodes; (b): 30 nodes

\section{CONCLUSION \& FUTURE WORKS}

This paper proposes an improvement of AODV routing protocol by adding the delay period of each path, together with number of hops, as a mixed metric that will be considered in route selection decision using fuzzy logic. The performance of the proposed scheme has been compared with the performance of the original AODV. The performance analysis showed that this fuzzy based multi constraint routing has better average end-to-end delay, packet delivery, and normalized routing load than the original routing algorithm. In future research study, other constraints can be taken to produce the fuzzy cost.

\section{REFERENCES}

[1] C. Siva Ram Murthy and B. S Manoj, "Ad Hoc Wireless Networks, Architecture and Protocols", Prentice Hall PTR, 2004.
[2] Charles E. Perkins, Pravin Bhagwat, "Highly dynamic destination sequenced distance vector routing (DSDV) for mobile computers", SIGCOMM '94: Proceedings of the Conference on Communications Architectures, Protocols and Applications, vol. 24, ACM Press, October 1994 , pp. 234-244.

[3] T. Clausen, P. Jacquet, "Optimized link state routing protocol (OLSR)", RFC 3626, IETF Network Working Group, October 2003.

[4] C. Parkins, E. Royer, and S. Das, Ad hoc onDemand Distance Vector (AODV) Routing, RFC3561, IETF,2003.

[5] D. Johnson, Y. Hu, and D. Maltz, " The Dynamic Source Routing Protocol (DSR) for Mobile Ad Hoc Networks for IPv4", RFC 4728, IETF, February 2007.

[6] Z. J. Hass and M. R. Pearlman, "The Performance of Query Control Schemes for the Zone Routing Protocol", IEEE/ACM Transactions on Networking (TON), 9:427-438, August, 2001.

[7] M. Joa-Ng and I. T. Lu, "A Peer-to-Peer ZoneBased Two-Level Link State Routing for Mobile Ad Hoc Networks", IEEE journal on Selected areas in Communications, vol. 17, no. 8, August 1999, pp. 1415- 1425.

[8] Hui Liu, Jie Li, Yan-Qing Zhang and Yi Pan, "An Adaptive Genetic Fuzzy Multi-path Routing Protocol for Wireless Ad-Hoc Networks", In the Proceedings of Sixth International Conference on Software Engineering, Artificial Intelligence, Networking and Parallel/Distributed Computing and First ACIS International Workshop on Self-Assembling Wireless Networks (SNPD/SAWN'05), SNPD 2005: pp. 468-475.

[9] A. Banerjee and P. Dutta, "Fuzzy-Controlled Route Discovery For Mobile Ad Hoc Networks", International Journal of Engineering Science and Technology, Vol. 2 (6), 2010, pp. 23472353.

[10] S. Marwaha, D. Srinivasan, K. T. Chen and A. Vasilakos, "Evolutionary fuzzy multi-objective routing for wireless mobile ad hoc networks", Congress on Eutionary Computation 2004, 2 (2004), pp. 1964 - 1971.

[11] C.C. Lee," Fuzzy logic in control systems: fuzzy logic controller-Part I and II", IEEE Transactions on Systems, Man, and Cybernetics 20 (2)(1990), pp. 404-418.

[12] Timothy J. Ross, "Fuzzy Logic with Engineering Applications", $2^{\text {nd }}$ Ed., Wiley, 2004.

[13] OMNeT++, http://www.omnetpp.org 